\title{
Collaboration in sensor network research: an in-depth longitudinal analysis of assortative mixing patterns
}

\author{
Alberto Pepe $\cdot$ Marko A. Rodriguez
}

Received: 16 September 2009/Published online: 31 December 2009

(C) The Author(s) 2009. This article is published with open access at Springerlink.com

\begin{abstract}
Many investigations of scientific collaboration are based on statistical analyses of large networks constructed from bibliographic repositories. These investigations often rely on a wealth of bibliographic data, but very little or no other information about the individuals in the network, and thus, fail to illustrate the broader social and academic landscape in which collaboration takes place. In this article, we perform an in-depth longitudinal analysis of a relatively small network of scientific collaboration $(N=291)$ constructed from the bibliographic record of a research centerin the development and application of wireless and sensor network technologies. We perform a preliminary analysis of selected structural properties of the network, computing its range, configuration and topology. We then support our preliminary statistical analysis with an in-depth temporal investigation of the assortative mixing of selected node characteristics, unveiling the researchers' propensity to collaborate preferentially with others with a similar academic profile. Our qualitative analysis of mixing patterns offers clues as to the nature of the scientific community being modeled in relation to its organizational, disciplinary, institutional, and international arrangements of collaboration.
\end{abstract}

Keywords Scientific collaboration networks - Sensor network and wireless research · Network evolution · Mixing patterns · Discrete assortativity · Homophily

\section{Introduction}

Scientific communities have large, well-established, and relatively well structured digital footprints which have increasingly been the focus of specialized research. These footprints, composed of scholarly publications and related artifacts, are employed for bibliometric

A. Pepe $(\bowtie)$

Center for Embedded Networked Sensing, University of California, Los Angeles, USA

e-mail: apepe@ucla.edu

M. A. Rodriguez

T-5, Center for Nonlinear Studies, Los Alamos National Laboratory, Los Alamos, USA

e-mail: marko@lanl.gov 
analyses involving coauthorship, citation, co-citation, acknowledgments, and other such indicators of scientific productivity and knowledge production. Coauthorship patterns are among the most studied scholarly and scientific phenomena. Recent studies of coauthorship have analyzed the literature production within specific domains such as high energy physics (Mele et al. 2006), genetic programming (Tomassini et al. 2007), neuroscience (Braun et al. 2001), nanoscience (Schummer 2004), library science (Liu et al. 2005), economics (Hollis 2001), and organizational science (Acedo et al. 2006). Similar analyses have also been comparative in nature and have explored social and normative differences of coauthorship behavior across different domains (Newman 2004a, b). Moreover, an increasing number of studies of this kind have accounted for the evolving component of scientific collaboration (Barabási et al. 2002, Catanzaro et al. 2004, Wagner and Leydesdorff 2005).

More specifically, coauthorship patterns have been widely and actively studied from a social network analysis perspective for over two decades (Fenner et al. 2007, Liberman and Wolf 1998, Lievrouw et al. 1987). Most social network research involved with coauthorship is based upon this underlying concept: two individuals (nodes) are regarded as coauthors if they appear together in the author list of a publication (edge). This relational structure works reasonably well when investigating coauthorship patterns in scholarly arrangements where publications are authored by relatively small groups. It is true that some scientific domains have experienced an increase in the number of authors per publication making it impossible to discern the nature and extent of individual contributions to a publication (Cronin 2005). A striking example of this phenomenon can be found in the domain of high-energy physics where author lists for a single publication often include tens or even hundreds of authoring researchers (Traweek 1992). For this reason, a number of recent studies of physics collaboration supplement traditional analytic techniques with more qualitative methods of survey research, i.e., directly asking authors to indicate the real nature of their contributions to a publication (Birnholtz 2006, Tarnow 2002). However, besides the singular case of high-energy physics, the vast majority of scholarly coauthorship networks incorporate collaboration circles of only a handful of authors per publication, suggesting that coauthorship activity can be adequately employed to construct a valid social network of collaboration (Newman 2004a, b).

In this article, we perform a temporal analysis of a coauthorship network constructed from the bibliographic record of a research center involved in the development and application of sensor network and wireless technologies. The network studied here is relatively small in size compared to networks generally analyzed in related research. The small size of the collaboration network results in a fundamental advantage: besides analyzing certain large-scale structural properties of the network, we can explore the social and academic arrangements in which collaboration patterns evolve, based on a set of node characteristics.

The study of node characteristics can provide insights into the level of "homophily" in a social network, i.e. the tendency of individuals to create ties with similar others [for a review, McPherson et al. 2001]. The homophily principle describes how homogeneous a network is in terms of specific sociodemographic, behavioral, or interpersonal characteristics. For example, a high level of homophily in a friendship network indicates that individuals with certain characteristics - such as race, ethnicity, political beliefs, and educational background - tend to make friends with individuals with similar characteristics. Many studies of homophily are grounded in sociology and investigate patterns of homophily as well as their driving forces and their implications. An established method to measure mathematically the level of homophily in a network is by computing its 
"assortativity" (also "assortative mixing"), i.e., the extent of mixing between similar nodes in a network (Newman 2003a, b). While many different components of similarity can be investigated, the vast majority of large-scale studies of networks look at the mixing of node degree, i.e. how nodes with similar degree preferentially attach to one another. Mixing patterns, however, can also be calculated based on discrete node-specific characteristics. In studies of scholarly and scientific collaboration, examples of characteristics that have been investigated include research interests (Havemann et al. 2006), academic domain (Moody 1963), geographical location (Lorigo and Pellacini 2007), age group (Bonaccorsi and Daraio 2003), and country of origin (Rodriguez and Pepe 2008) of the individuals in the network. Studying networks in terms of these node properties can offer insights into the mechanisms by which disciplinary, institutional, and spatial arrangements shape, and are shaped by, collaboration patterns.

The present study, exploratory in nature, ties a quantitative analysis of a network's assortativity structure to a qualitative explanation of the social and academic landscape in which such network is embedded. Scholars working with scientific collaboration networks are increasingly becoming interested in grounding their quantitative analyses in sociological theory. An example is the work of Kretschmer and Kretschmer (2007) that investigates whether the distribution of co-author pairs' frequencies in a collaboration network can be regarded as a "social Gestalt". They derive a mathematical function to describe social Gestalts in networks and apply it on four large scale coauthorship networks (cumulative $N_{i j}>2 M$ co-author pairs) to explore the relationship between coauthorship and node-based author productivity.

This study focuses on a much smaller collaboration network $(N=291)$. The small size of the network allows us to both perform a quantitative analysis of selected structural properties of the network and provide a a sociological explanation of our findings, based on an in-depth qualitative analysis of assortative mixing patterns. We demonstrate how certain social and academic dynamics, for example the emergence of new international collaborations or the inception of new inter-departmental efforts, have varying levels of control in the resulting topology and configuration of the scientific collaboration network.

\section{Present study}

This article presents the findings of a study of scientific collaboration at the Center for Embedded Networked Sensing (CENS). ${ }^{1}$ CENS is a National Science Foundation (NSF) Science \& Technology Center funded in 2002. CENS supports interdisciplinary collaborations among faculty, students, and staff of five partner universities in Southern California: University of California, Los Angeles (UCLA); University of Southern California (USC); University of California, Riverside; California Institute of Technology (Caltech); and University of California, Merced. From 2005, CENS features a headquarter office base located within Boelter Hall at UCLA.

The mission of CENS research is to use sensor network technology to reveal previously unobservable phenomena. From its inception, CENS has developed and deployed sensor network devices for the study of a wide range of natural phenomena, such as seismic activity, fluid contaminant transport, and bird breeding behavior. Besides these pursuits in the natural sciences, the social and built environments have progressively become the focus

1 The website of the Center for Embedded Networked Sensing (CENS) is available online at http://www.research.cens.ucla.edu/. 
of applied CENS research: sensing mobile systems are being employed for the study of public health, environmental protection, urban planning, and cultural expression.

The type of research conducted at CENS now spans a wide spectrum of disciplines and applications (from biology to seismology, from wireless telecommunications to statistics, from education to environmental science) requiring continuous cooperation among individuals that, otherwise, would probably not interact beyond the walls of traditional university departments and faculties. In such a diverse scholarly and scientific landscape, distributed collaboration on multi-disciplinary subjects constitute a fundamental leverage for scientific research.

\section{Data collection}

Computing the range, population and configuration of an interdisciplinary, multi-institutional research center like CENS can be an arduous task. In the case presented in this article, these difficulties have to do with the inherently open and dynamic nature of modern science research centers. Unlike other types of organizational arrangements for which the boundaries are more or less evident (e.g. academic institutions and departments, corporate and government centers), many modern research centers and laboratories act as umbrella organizations with very flexible and blurry boundaries. CENS, for example, includes researchers from multiple institutions and disciplines. CENS scholars seamlessly interact with each other within and beyond institutional and departmental boundaries: collaboration patterns are ubiquitous and non-uniformly distributed. Researchers affiliated with CENS may also be affiliated with other research laboratories and perform interdisciplinary work on other projects and under different affiliations. Moreover, many CENS collaborations include researchers that are not officially affiliated with the center. In other words, the nature and extent of contribution to CENS collaboration depends on a number of organizational and scholarly factors, and is not solely restricted to individuals officially affiliated with the center. Under these conditions, what is the best way to construct a network that accurately captures scientific collaboration of this research center?

Previous environment-specific studies of collaboration have delineated the population under study by relying on publication data contained in an institutional repository (Acedo et al. 2006) or domain-specific bibliographic databases (Liu et al. 2005) to mine patterns of coauthorship that take place within a given institution or academic domain, respectively. For the purpose of this article, we used a similar mechanism, thus including in our population not only CENS members, but also individuals that though not officially affiliated, have contributed to the production of CENS or CENS-related scholarly publications.

We constructed a database of publications by assembling the items included yearly in the NSF Annual Reports, which contain the official list of documents published by CENS for a given fiscal year. ${ }^{2}$ For every publication in the database, we collected author names and publication dates (years) to construct a coauthorship network, i.e. a network consisting of individuals (nodes) that are connected to each other (via edges) if they are recorded as authors on the same scholarly publication. The resulting bibliographic dataset consisted of 547 manuscripts (370 conference proceedings, 159 journal articles, 17 book chapters and 1 book), published over a period of 7 years (2001-2007).

This bibliographic database was used to generate a weighted undirected network in which nodes represent authors and edges represent coauthorship activity among them. For example, if the present paper had to be included in this network, its authors (Pepe and Rodriguez)

\footnotetext{
${ }^{2}$ CENS Annual Reports are available online at http://www.research.cens.ucla.edu/about/annual_reports/.
} 
would become two distinct nodes, connected by an edge. In order to determine the weights between nodes, i.e. the strength of collaboration among coauthors, we used a weighting mechanism proposed by Newman (2001) by which the weight of the edge between nodes $i$ and $j$ is:

$$
w_{i j}=\sum_{k} \frac{\delta_{i}^{k} \delta_{j}^{k}}{n_{k}-1},
$$

where $\delta^{k}{ }_{i}$ is 1 if author $i$ collaborated on paper $k$ (and zero otherwise) and $n_{k}$ is the number of coauthors of paper $k$. For the example above, the edge between authors Pepe and Rodriguez would have $w_{i j}=1$. An article written by three authors (e.g., Pepe, Rodriguez, and Bollen) would result in three edges (Pepe-Rodriguez, Pepe-Bollen, and RodriguezBollen), each one with $w_{i j}=0.5$. And so on. As such, this weighting mechanism confers more weight to small and frequent collaborations, based on the assumptions that: (i) publications authored by a small number of individuals involve stronger interpersonal collaboration than multi-authored publications, and (ii) authors that have authored multiple papers together know each other better on average and thus collaborate more strongly than occasional coauthors (Newman 2001).

The resulting network data were "sliced" according to publication year yielding to 7 separate networks, each one representing the cumulative collaborative effort of CENS researchers up to that year.

Network range, configuration, and topology

For each one of the coauthorship networks under study, for years 2001 through 2007, we calculated some fundamental network statistics, presented in Table 1.

An analysis of these statistics provides insights into the evolution of the CENS coauthorship network over time. The first three rows of the table contain, for every year in the period under study, the cumulative number of (a) authors, i.e. nodes in the network, (b) publications (journal articles, conference papers, etc.), and (c) collaborations (coauthoring events), i.e. edges in the network. When analyzed over time, these three values all follow a similar trend, which highlights two distinct time periods: a first term (2001-2004) during

Table 1 A summary of the fundamental network statistics of the CENS coauthorship network for the time period 2001-2007: number of authors, publications and collaborations (range), number of connected components and diameter of the largest connected component (configuration), and average path length, clustering coefficient and degree assortativity (topology)

\begin{tabular}{llllllll}
\hline Quantity/year & 2001 & 2002 & 2003 & 2004 & 2005 & 2006 & 2007 \\
\hline Authors (nodes) & 35 & 68 & 127 & 203 & 228 & 278 & 291 \\
Publications & 23 & 69 & 175 & 303 & 418 & 496 & 547 \\
Collaborations (edges) & 182 & 346 & 690 & 1248 & 1598 & 2158 & 2536 \\
Connected components & 4 & 5 & 8 & 13 & 9 & 6 & 5 \\
Diameter & 3 & 4 & 6 & 7 & 8 & 8 & 7 \\
Average path length, $\ell$ & 1.543 & 2.324 & 3.090 & 3.038 & 3.339 & 3.224 & 2.944 \\
Clustering coefficient, $C$ & 0.645 & 0.543 & 0.432 & 0.387 & 0.389 & 0.337 & 0.329 \\
Degree assortativity, $r$ & 0.432 & 0.299 & 0.272 & 0.187 & 0.180 & 0.166 & 0.165 \\
\hline
\end{tabular}

All values presented are cumulative 
which all values increase sharply (roughly doubling in size from year to year), and a second term (2004-2007), during which the growth slows down. In particular the author count values indicate that CENS quickly became large and diversified in its population in the first term reaching a solid population base of collaborators by the year 2004. In the second term, from 2004 to 2007, the author population increased again, but to a much lesser extent (from 203 to 291 individuals), while the number of published works and collaborations maintained a regular growth (from 1248 to 2536 collaborations), suggesting the formation of a core CENS authoring base.

This finding is confirmed by a quick analysis of the network's configuration. The number of connected components, i.e. the number of maximal connected subgraphs, goes from 4, in 2001, to 13, in 2004, indicating that the network becomes more fragmented in the first term, even if collaboration is overall increasing. In the second term, however, the number of connected components drops and the network quickly solidifies into a giant component, which indicates a solid base of strong collaboration. By looking at the the network diameter, i.e. the length of the longest geodesic path in the largest connected component, the formation of the giant component in year 2004 becomes evident. This is further reinforced by a quick analysis of Table 2, which lists component populations by year.

The preliminary analysis of these first two sets of values from Table 1 gives us a good understanding of the evolution of the range and configuration of the network over time. A third set of values, presented in Table 1 (average path length, clustering coefficient, and degree assortativity) can be investigated to provide an in depth understanding of its topology.

The average path length is the average length of the shortest paths between all possible node pairs and, in turn, an indicator of the efficiency of information transfer in a social network (Wasserman and Faust 1994). Short average path length, and thus high information transmission, are typical characteristics of many real and small-world networks (Watts and Strogatz 1998). In the network under study, the average path length is about 1.5 in 2001; it grows steadily in the first term, reaching a value of about 3.0, which stays roughly constant throughout the second term. This indicates that once the CENS authoring base is formed, an average of 3 steps are necessary to transfer information among any two pairs of nodes. Remarkably, this value resembles more closely that found in typical smallworld networks, such as movie actors $(\ell=3.65)$ (Watts and Strogatz 1998), than that of scholarly coauthorship networks, such as mathematics $(\ell=9.5)$ and neuroscience $(\ell=6)$ (Barabási et al. 2002); yet, this relatively low average path is due to the relatively small size of the network analyzed (the mathematics and neuroscience coauthorship networks have $N=70,975$ and 209, 293, respectively).

Table 2 Node populations of connected components, by year

\begin{tabular}{|c|c|c|}
\hline Year & \# & Population \\
\hline 2001 & 4 & 18674 \\
\hline 2002 & 5 & 3816842 \\
\hline 2003 & 8 & 9310754332 \\
\hline 2004 & 13 & 1501088543333222 \\
\hline 2005 & 9 & 20553333222 \\
\hline 2006 & 6 & 26254322 \\
\hline 2007 & 5 & 2804322 \\
\hline
\end{tabular}


The clustering coefficient measures the density of clique-like triangles in a network. High clustering coefficient coupled with short average path length indicates that a network exhibits small-world properties (Newman 2003a, b). In the CENS coauthorship network, the clustering coefficient decreases steadily over time, from an initial value of 0.645 in 2001, to 0.329 in 2007. This suggests that the network becomes less cliquish and collaboration patterns becomes more uniform across the network over time. This trend reveals that the CENS network initially matches the typical topology of highly-clustered disciplines such as physics $(C=0.56)$ and biology $(C=0.6)$ (Newman 2003a, b) but later drops to the values normally recorded in less cliquish domains, such as mathematics $(C=0.34)$ (Grossman and Ion 1995).

A final indicator of network topology presented here is degree assortativity (Newman 2002). In the network presented here, degree-based assortativity decreases steadily over time from a value of 0.432 in 2001 to 0.165 in 2007 . Interestingly, the decline of the degree assortativity measure follows very closely that of the clustering coefficient- the correlation between the two is $\rho=0.964$ ( $p$-value $<0.005$ ). This means that as collaboration patterns in the network become more sparse and uniform (decreasing $C$ ), they also become more mixed (decreasing $r$ ), i.e. highly-connected individuals begin to collaborate with lowlyconnected ones. In the next section, we extend the study of assortativity to a set of discrete characteristics, namely authors' academic department, affiliation, position, and country of origin. Analysis of these mixing patterns allows us to understand the homophilious and heterophilious components that contribute to the observed growth of the network.

\section{Analysis of network evolution by discrete assortative mixing}

The preliminary analysis of the CENS coauthorship network presented in the previous section reveals the following scenario. In 2001, the network of collaboration is small and very fragmented. During the first few years of activity, however, the CENS group grows significantly in the number of authors and collaborations. By the end of 2004, a solid base of collaborating authors (i.e. a giant component) is formed. In the analyzed network, smallworld effects become less prominent over time; in particular, average distance between individuals becomes larger (increasing $\ell$ ), and collaboration patterns in the network become more sparse (decreasing $C$ ) and more mixed (decreasing $r$ ).

Although our preliminary analysis presents a fairly comprehensive account of the range, configuration and topology of the studied network of scientific collaboration over time, we believe that it fails to provide a sociological explanation of the dynamics underlying the observed patterns. In particular, we were curious to explore further the correlation between clustering coefficient and assortativity. Our preliminary analysis indicates that there exists a solid link between these two patterns: (a) the network becoming more sparse and uniform, and (b) collaboration patterns becoming more mixed. However, this analysis is restricted to degree assortativity and thus ignores other mixing patterns that might have contributed to the decrease in network clustering over time. For this reason, we became interested in deepening our understanding of the sociological and academic context of the CENS collaboration network to identify specific patterns that might account for the observed clustering pattern. For example, can we speculate that the network becoming more sparse is indicative of higher interdisciplinary collaboration and/or higher collaboration across different institutions? In this context, the question that we would like to address is: what specific mixing patterns are accountable for the decrease in the network's clustering coefficient? 
In order to address this question, we collected this additional information relative to each author in the network under study: (a) academic affiliation, (b) academic department, (c) academic position, and (d) country of origin. We collected these metadata via manual techniques, i.e. gathering required information on the authors' personal web pages and consulting online directories from university and department web sites. It is worth noting that all the parameters collected (except for country of origin) are subject to change over time, even in the short timespan studied in this article. Researchers and scientists might change institution, department and position in a 7-year time period. For this reason, we consulted not only authors' personal web sites, but also their curriculum vitae and biographies to record changes in their academic affiliation, department and position. Curriculum vitae were also useful to collect authors' country of origin, which, for the purpose of this study, we consider to be the country in which individuals pursued their high-school education.

The temporal analysis of degree assortativity, presented in the previous section, indicates the extent to which individuals in the network co-author preferentially to other individuals with similar degree centrality. Using the newly collected author metadataacademic affiliation, department, position and country of origin-we can extend our investigation of assortativity to compute mixing patterns based on these discrete parameters. In our case, all analyzed parameters are nominal and we can thus measure discrete assortativity coefficient, $r$, using the following formula (Newman 2003a, b):

$$
r=\frac{\sum_{i} e_{i i}-\sum_{i} a_{i} b_{i}}{1-\sum_{i} a_{i} b_{i}}
$$

where $e_{i j}$ is the fraction of edges in a network that connect a node of type $i$ to one of type $j$, $a_{i}$ is the fraction of edges that have a node of type $i$ on the head of the edge, and $b_{i}$ is the fraction of edges that have a node of type $i$ on the tail of the edge. Finally, $r=-1$ when there is perfect disassortative mixing, $r=0$ when there is no assortative mixing, and $r=1$ when there is perfect assortative mixing. In other words, the discrete assortativity coefficient, $r$, indicates the level of homophily of the network for a certain parameter. For example, if $r$ for academic affiliation is 1.0, this means that individuals in the network only write papers with other individuals with same institutional affiliation. In this kind of network, there are no multi-institutional collaborations. On the other side of the spectrum, we can imagine a completely disassortative network $(r=-1)$ in which every single collaboration (i.e. paper) in the network is authored by individuals that belong to different institutions.

Table 3 presents the discrete assortativity coefficients for the network under study based on authors' academic affiliation, department, position and country of origin, calculated at seven temporal snapshots of the network (2001 through 2007). A visual representation of these values is also presented in the plot of Fig. 1.

Table 3 Discrete assortativity coefficients (2001-2007) based on authors' academic affiliation, department, position and country of origin

\begin{tabular}{llllllll}
\hline Property/year & 2001 & 2002 & 2003 & 2004 & 2005 & 2006 & 2007 \\
\hline Academic affiliation & 0.438 & 0.448 & 0.501 & 0.533 & 0.550 & 0.584 & 0.544 \\
Academic department & 0.463 & 0.535 & 0.574 & 0.560 & 0.555 & 0.516 & 0.474 \\
Academic position & 0.177 & 0.192 & 0.188 & 0.184 & 0.182 & 0.177 & 0.177 \\
Country of origin & 0.245 & 0.286 & 0.369 & 0.350 & 0.347 & 0.352 & 0.367 \\
\hline
\end{tabular}




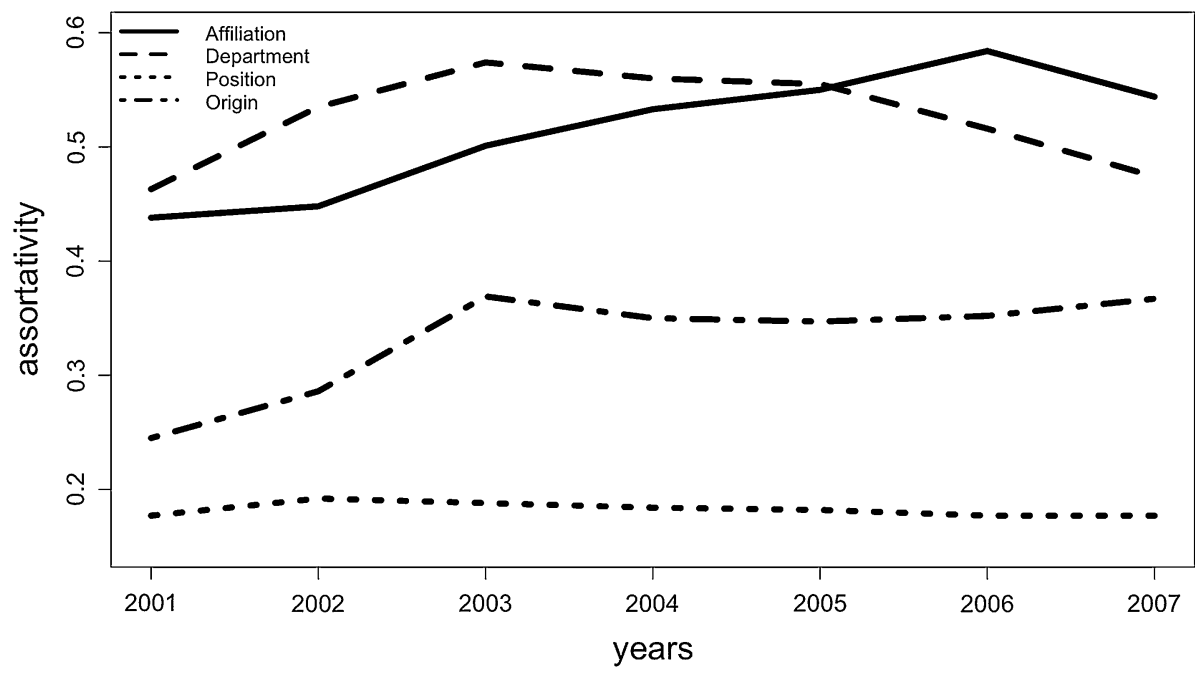

Fig. 1 Plot of discrete assortativity coefficients (2001-2007) based on authors' academic affiliation, department, position and country of origin

Looking at Fig. 1, it is easy to deduce that these assortativity coefficients are all within the same range-between a minimum of 0.1 and a maximum of 0.6 . Also, they do not change very much over the period under study-fluctuations in the 7-year period rarely exceed 0.1. Overall, the network is more assortative by academic affiliation and department, and less assortative by academic position and country of origin. In particular, academic position has very little influence on the overall topology of the CENS coauthorship network, compared to the other computed measures. Assortativity by academic position never exceeds a value 0.2 and, very importantly, it remains practically unchanged throughout the period under study. For this reason, we do not present below a detailed analysis of assortative mixing by academic position. We investigate, however, the extent and nature of the other three mixing patterns in the coauthorship network: academic affiliation, department and country of origin. In the remainder of this section we present, for each one of these characteristics, a detailed interpretation of our findings. Our aim is to push our understanding of the assortativity coefficients further, decomposing the observed collaboration patterns along specific components, to allow a more in-depth temporal analysis of the observed mixing patterns.

\section{Academic affiliation}

From Fig. 1, the assortativity coefficient based on nodes' academic affiliation grows steadily over time, by about 0.1 , from 0.438 in 2001 to 0.544 in 2007 . This indicates that, overall, authors in the CENS coauthorship network have increasingly collaborated with other authors from the same institutional affiliation, in the time period under study. In the latest snapshot of the CENS network (year 2007) academic affiliation is the single most assortative characteristic, suggesting that CENS authors collaborate preferentially with individuals in their institution. This finding matches an earlier observation that the community structure of CENS collaboration matches very closely its institutional configuration (Rodriguez and Pepe 2008). 


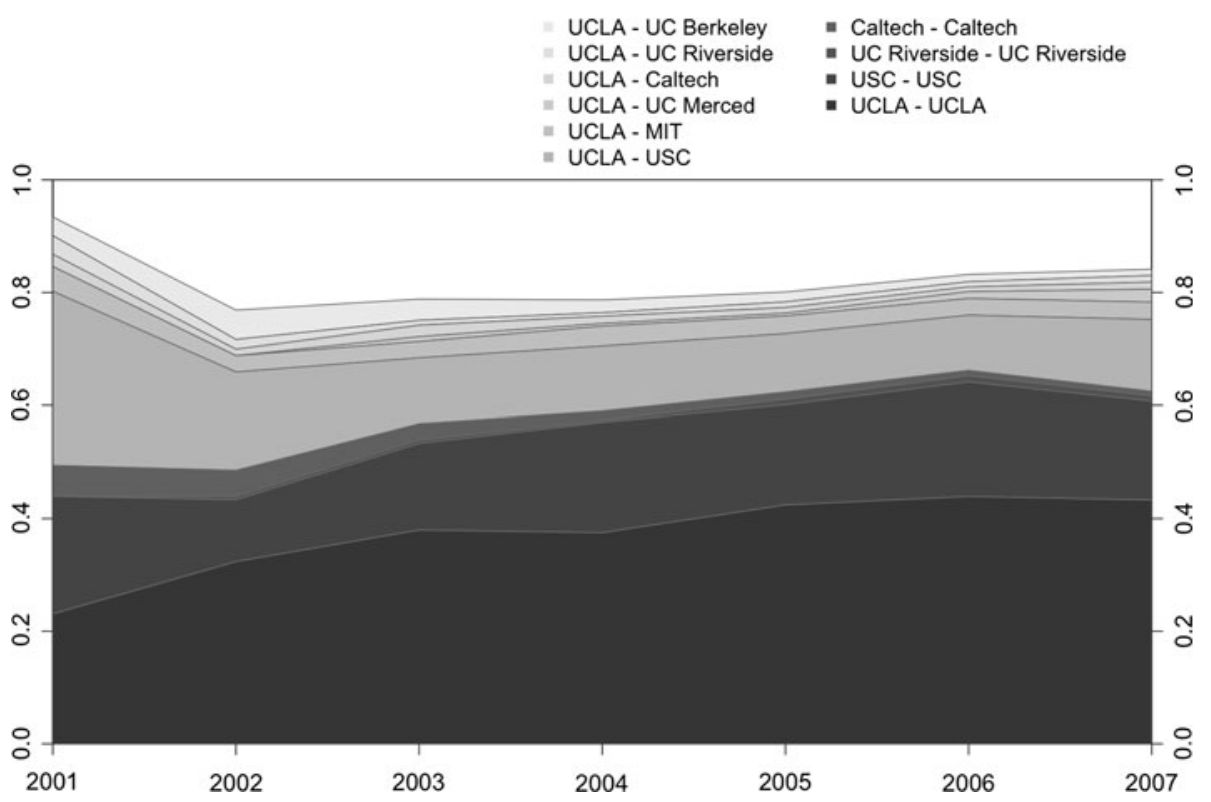

Fig. 2 Top ten most recurrent academic affiliation pairs as fraction of total volume of collaboration. Darker polygons at the bottom are intra-institutional collaboration, while lighter polygons depict inter-institutional collaboration

We would like to investigate this finding further, analyzing the specific intra- and interinstitutional collaborations that contributed to making the network more assortative over time. In Fig. 2 we present as a stacked plot the most recurrent mixing patterns (affiliation pairs) as a fraction of the total volume of collaborations each year. The bottom four rows of Fig. 2 list the institutional pairs contributing to intra-institutional collaboration (e.g., UCLA-UCLA), whereas the top six rows present the pairs contributing to inter-institutional collaboration (e.g., UCLA-USC).

The stacked plot of Fig. 2 allows us to decompose the assortativity coefficient trend lines of Fig. 1 for discrete parameter academic affiliation. From Fig. 1, assortativity by affiliation increases steadily from 2001 to 2006 and finally drops slightly from 2006 to 2007. This trend can be understood in terms of the growth of intra- and inter-institutional collaborations, presented in Fig. 2. From the stacked plot of Fig. 2, we note that in 2001, the vast majority of recorded collaborations involves intra- and inter-institutional efforts between UCLA and USC individuals. In year 2007, the picture is not very different, with UCLA and USC still composing the bulk of the total volume of collaborations. However, a closer look at the components of the plot reveals that intra-institutional collaboration at UCLA has doubled in volume (from 0.2 to 0.4 ) while inter-institutional collaboration (UCLA-USC) has halved (0.3-0.15), compared to 2001 values. USC-USC collaboration stays roughly constant throughout the period under study. The increase in UCLA-UCLA and the decrease of UCLA-USC collaborations are the components that are most responsible for the increase in assortativity coefficient by affiliation from 2001 to 2007, presented in Fig. 1.

There are some other collaboration dynamics that contribute to this trend. For example, besides UCLA-USC, the overall inter-institutional effort of UCLA decreases (e.g. 
collaborations with UC Berkeley and UC Riverside). Moreover, intra-institutional collaborations by Caltech researchers (which make almost $10 \%$ of the total volume in 2001) fade away over time. In sum, by year 2007, the collaboration scenario at CENS is largely dominated by publications authored within UCLA. Based on this finding, we can conclude that despite CENS's mission to be a multi-institutional research center, the temporal decomposition of coauthorship patterns demonstrates that CENS collaboration became less inter-institutional from 2001 to 2007 consolidating around its main institution, UCLA. The steady increase in UCLA-UCLA collaboration can possibly be attributed to the construction of a CENS headquarter office at UCLA, completed in 2005. We can speculate that the CENS headquarter has brought UCLA scholars closer to each other, enabling interpersonal collaboration among them and, in turn, boosting coauthorship activity. ${ }^{3}$

\section{Academic department}

From Fig. 1, the assortativity coefficient for academic department has the following trend. In year 2001, the CENS network is heavily assortative based on academic department $(r=0.463)$. In the following two years, assortativity increases even more, reaching a peak of 0.574 in 2003. This means that in 2003, the CENS coauthorship was very highly fragmented by department. By extension, we can speculate that at this time, collaboration patterns were vastly mono-disciplinary. In later years, however, assortativity by department decreases. Even though the value recorded in 2007 ( $r=0.474)$ is roughly equivalent to the network's outset, the trend observed from 2003 to 2007 indicates the CENS collaboration network becoming more interdisciplinary. A decomposition of the observed coauthorship patterns can be obtained by inspecting the stacked plot of Fig. 3 that depicts the most recurrent mixing pattern pairs by academic department as fraction of total volume of collaboration.

At the network's outset, CENS collaboration is dominated by intra-departmental collaborations in Computer Science and Electrical Engineering. The most prominent interdepartmental collaborations are between Computer Science and both Biology and Information Sciences. The increase in the assortativity coefficient by department from 2001 to 2003 (shown in Fig. 1) can be attributed to a number of factors, including (i) a slight increase in collaborations among Electrical Engineers, (ii) the appearance of novel collaborations among Biologists, and (iii) a substantial drop in collaborations by Computer Scientists with both Biologists and Information Scientists.

In the long run, however, the intra-departmental volume of collaboration among Computer Scientists decreases steadily over time. This decrease, coupled with the growth of a number of inter-departmental collaborations (Computer Science with Electrical and Civil Engineering, as well as Electrical Engineering with Biology and Statistics), is most responsible for the assortativity coefficient trend presented in Fig. 1, i.e., the CENS coauthorship network becomes less assortative by department, and thus more inter-disciplinary, over time.

The observed patterns can be interpreted as follows. First the overall presence of intradepartmental collaborations in Computer Science is telling of the nature of research being performed at CENS. The domain of networked sensing emerges historically from computer network research and is thus, normally located as a branch in departments of Computer Science. Sensor network technologies, however, require the design and construction of

\footnotetext{
3 This is a fair assumption especially for the authoring of scientific conference papers, that have a much quicker publication turnaround than journal articles.
} 


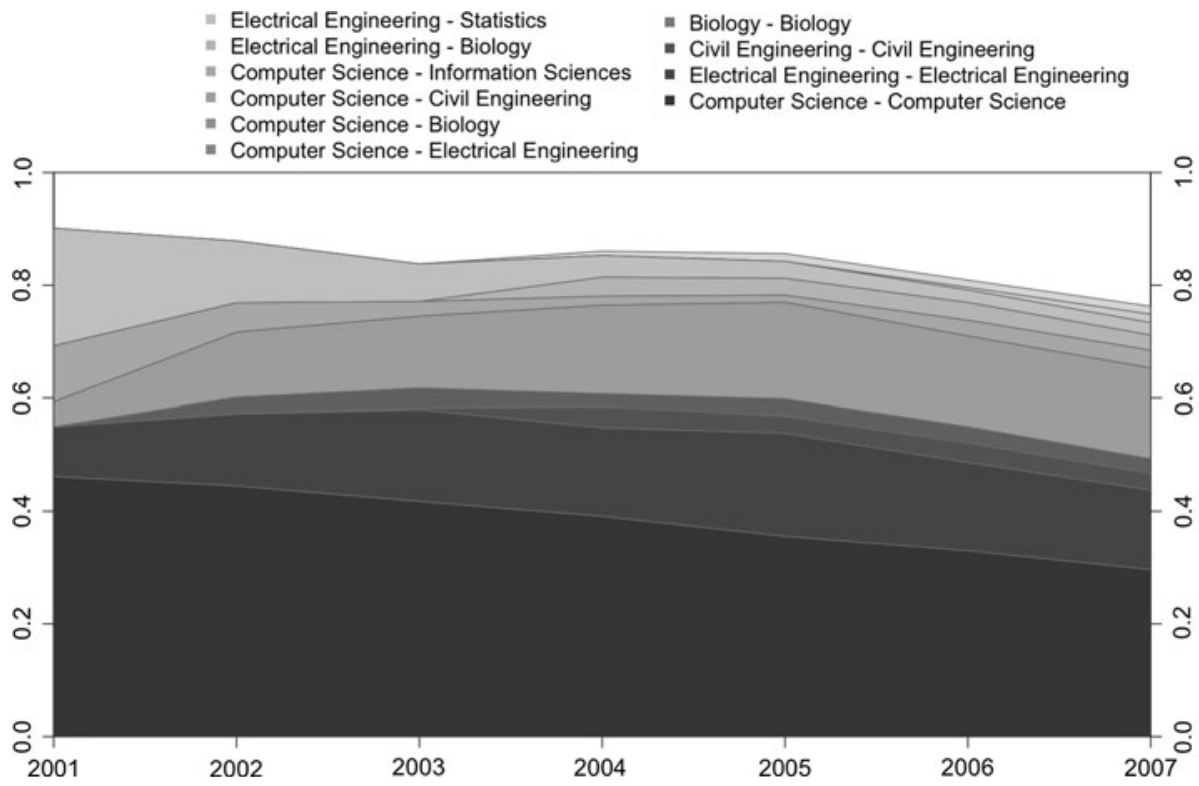

Fig. 3 Top ten most recurrent academic department pairs as fraction of total volume of collaboration. Darker polygons at the bottom are intra-departmental collaboration, while lighter polygons depict interdepartmental collaboration

wireless sensors, and, in turn, interaction between computer sciences and engineering disciplines follows necessarily. This growing incidence of a core set of Electrical Engineering collaboration (both intra- and inter-departmental) is evident in Fig. 3. It is interesting to note that inter-departmental collaborations with Electrical Engineers involve not only Computer Science, but also Biology (a major scientific application area for sensor networks) and Statistics (a discipline increasingly required by field scientists to deal with issues related to sensor data cleaning, analysis, and modeling). Finally, it is worth noting that the volume of intra- and inter-departmental collaborations involving the department of Civil Engineering increases over time, possibly reflecting the inception in 2004 of a new application area at CENS involved in the development and application of sensing technologies in urban and social settings.

In sum, CENS, a research center emerged as a sub-domain of Computer Science, has progressively become more inter-disciplinary over time. The increase in inter-disciplinarity can be attributed to CENS' need to develop sensor network technologies (Electrical Engineering), apply and deploy them in field environments (Biology and Civil Engineering), and concurrently deal with data analysis issues (Statistics).

\section{Country of origin}

The final discrete assortativity coefficient we analyze in this article is the country of origin of the individuals in the network. Figure 1 shows that this coefficient increases steadily in the first two years and then levels off in later years at a value around $r=0.35$. What specific intra- and inter-national collaborations may account for such trend? Even if the vast majority of publications analyzed in this study are based on research performed in the United States, it is interesting to explore the tendency of individuals to collaborate with 


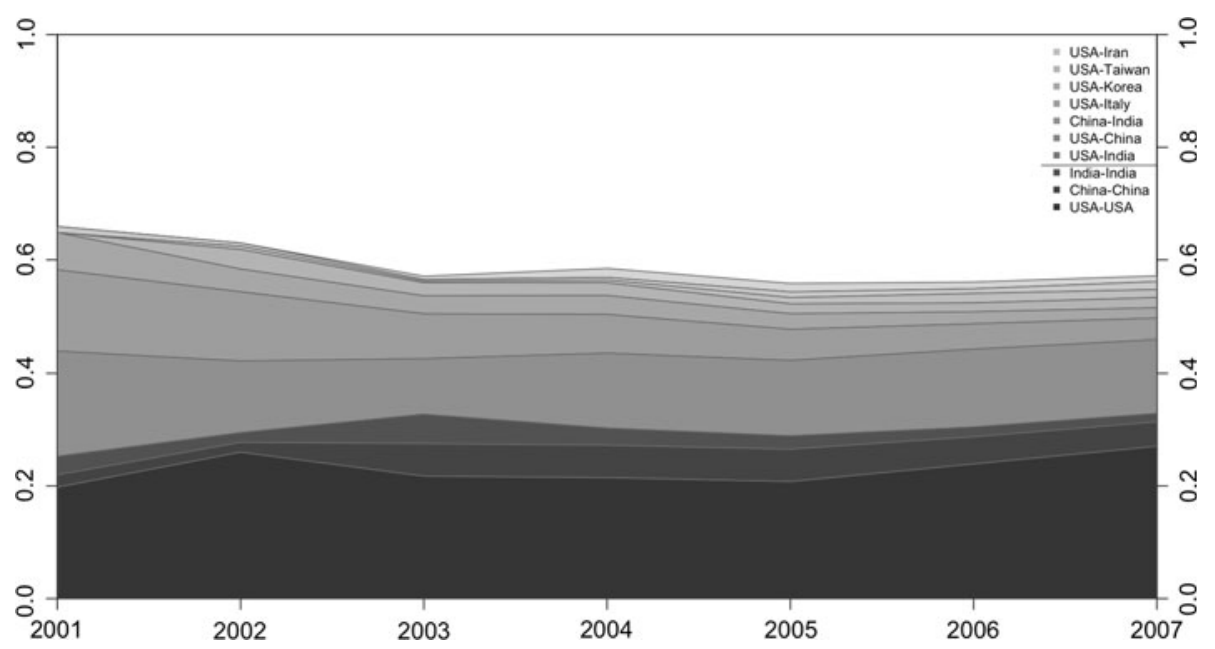

Fig. 4 Top ten most recurrent country of origin pairs as fraction of total volume of collaboration. Darker polygons at the bottom depict intra-national collaboration, while lighter polygons depict inter-national collaboration

others from their country of origin, even when they are working and living abroad, or in different countries. We present in Fig. 4 the ten most recurrent mixing patterns by country as fraction of yearly cumulative volume of collaboration.

At the network's outset, the vast majority of collaborations is among Americans and between Americans and Indian and Chinese researchers. By year 2003, the picture only changes slightly. More intra-national collaborations appear (China-China and IndiaIndia), while inter-national collaborations between USA, India and China drop. These dynamics account for the growth of overall assortativity by country of origin recorded from 2001 to 2003, and visible in Fig. 1. By year 2007, the picture becomes more variegated. Intra-national collaborations among American researchers still dominate. However, a number of novel inter-national collaborations emerge, namely between USA and Italy, South Korea, and Iran. These observations reveal that as soon as CENS acquired a solid research core of collaboration, by year 2004, the center began to attract and involve collaborations by international scholars.

\section{Conclusion}

A great deal of research on scientific collaboration is performed on large-scale networks, constructed from bibliographic data harvested from domain-based and institutional document repositories. While these analyses rely on great quantities of data to study the structure, evolution and similar macroscopic features of scientific collaboration patterns, they often ignore certain contextual and microscopic factors, such as the social and academic arrangements in which collaboration takes place. This is because many available bibliographic datasets contain detailed publication metadata, but very little or no data about the authors writing those publications.

In this article, we perform a longitudinal analysis of the range, configuration and topology of a small network of scientific collaboration over a 7-year period. The network 
presented here is constructed from the bibliographic record of CENS, a research center involved in the development and application of sensor network technologies. Given the relatively small size of the network $(N=291$, in its largest year), we were able to manually collect additional metadata for every individual in the network studied. We used these node characteristics to explore the assortative mixing based on academic department, affiliation, position, and country of origin.

Our findings reveal that, in the period under study, the CENS collaboration network: (a) becomes more assortative in terms of academic affiliation, i.e. more intra-institutional, (b) becomes less assortative in terms of academic department, i.e. more inter-disciplinary, (c) is not assortative in terms of academic position, i.e. collaboration patterns are not dependent on researchers' academic positions, and (d) is only slightly assortative in terms of country of origin, i.e. the extent of inter-national collaboration decreases slightly over time.

We interpreted our findings in terms of the specific components that constitute these mixing patterns, finding that (i) the increase in intra-institutional collaboration is possibly caused by CENS research consolidating around its headquarter base at UCLA, completed in 2004; (ii) the increase in inter-disciplinarity is largely due to the shift a CENS' research agenda, to incorporate new domains, such as civil engineering and urban planning, besides the domains traditionally associated with sensor network research, i.e., computer science and electrical engineering; and (iii) the volume of international collaboration between USA, India, and China decreased but new smaller international efforts began as the organization matured.

This qualitative explanation of our findings revealed specific small-scale patterns that a quantitative analysis of assortativity alone would have failed to uncover. We speculate that supporting social network analyses with the proposed qualitative investigation of mixing patterns can provide a deeper understanding of the dynamics that shape (and are in turn shaped) by the changing socio-academic landscape in which scientific collaboration takes place.

Open Access This article is distributed under the terms of the Creative Commons Attribution Noncommercial License which permits any noncommercial use, distribution, and reproduction in any medium, provided the original author(s) and source are credited.

\section{References}

Acedo, F., Barroso, C., Casanueva, C., \& Galan, L. (2006). Co-authorship in management and organizational studies: An empirical and network analysis. Journal of Management Studies, 43(5), 957-983.

Barabási, A. L., Jeong, H., Neda, Z., Ravasz, E., Schubert, A., \& Vicsek, T. (2002). Evolution of the social network of scientific collaborations. Physica A, 311(3-4), 590-614.

Birnholtz, J. P. (2006). What does it mean to be an author? The intersection of credit, contribution, and collaboration in science. Journal of the American Society for Information Science \& Technology, 57(13), 1758-1770.

Bonaccorsi, A., \& Daraio, C. (2003). Age effects in scientific productivity. Scientometrics, 58(1), 49-90.

Braun, T., Glanzel, W., \& Schubert, A. (2001). Publication and cooperation patterns of the authors of neuroscience journals. Scientometrics, 51(12), 499-510.

Catanzaro, M., Caldarelli, G., \& Pietronero, L. (2004). Social network growth with assortative mixing. Physica A Statistical Mechanics and its Applications, 338, 119-124.

Cronin, B. (2005). The hand of science. Lanham: Scarecrow Press.

Fenner, T., Levene, M., \& Loizou, G. (2007). A model for collaboration networks giving rise to a power-law distribution with an exponential cutoff. Social Networks, 29(1), 70-80.

Grossman, J. W., \& Ion, P. D. F. (1995). On a portion of the well-known collaboration graph. Congressus Numerantium, 108, 129-131. 
Havemann, F., Heinz, M., \& Kretschmer, H. (2006). Collaboration and distances between German immunological institutes-a trend analysis. Journal of Biomedical Discovery and Collaboration 1(1), 6.

Hollis, A. (2001). Co-authorship and the output of academic economists. Labour Economics, 8(28), 503-530.

Kretschmer, H., \& Kretschmer, T. (2007). Lotka's distribution and distribution of co-author pairs' frequencies. Journal of Informetrics, 1(4), 308-337.

Liberman, S., \& Wolf, K. B. (1998). Bonding number in scientific disciplines. Social Networks, 20(3), 239-246.

Lievrouw, L. A., Rogers, E. M., Lowe, C. U., \& Nadel, E. (1987). Triangulation as a research strategy for identifying invisible colleges among biomedical scientists. Social Networks, 9, 217-248.

Liu, X., Bollen, J., Nelson, M. L., \& Van de Sompel, H. (2005). Co-authorship networks in the digital library research community. Information Processing \& Management, 41(6), 1462-1480.

Lorigo, L., \& Pellacini, F. (2007). Frequency and structure of long distance scholarly collaborations in a physics community. Journal of the American Society for Information Science \& Technology, 58(10), 1497-1502.

McPherson, M., Smith-Lovin, L., \& Cook, J. M. (2001). Birds of a feather: Homophily in social networks. Annual Review of Sociology, 27, 415-444.

Mele, S., Dallman, D., Vigen, J., \& Yeomans, J. (2006). Quantitative analysis of the publishing landscape in high-energy physics. Journal of High Energy Physics, 12, 1-23.

Moody, J. (2004). The structure of a social science collaboration network: Disciplinary cohesion from 1963 to 1999. American Sociological Review, 69(26), 213-238.

Newman, M. E. J. (2001). Scientific collaboration networks. II. Shortest paths, weighted networks, and centrality. Physical Review E, 64, 016132.

Newman, M. E. J. (2002). Assortative mixing in networks. Physical Review Letters, 89(20).

Newman, M. E. J. (2003a). Mixing patterns in networks. Physical Review E, 67(2), 026126.

Newman, M. E. J. (2003b). The structure and function of complex networks. SIAM Review, 45, 167.

Newman, M. E. J. (2004a). Who is the best connected scientist? A study of scientific coauthorship networks. In: Ben-Naim, E., Frauenfelder, H., \& Toroczkai, Z. (eds) Complex networks (pp. 337-370). Berlin: Springer.

Newman, M. E. J. (2004b). Coauthorship networks and patterns of scientific collaboration. Proceedings of the National Academy of Sciences, 101(Suppl 1), 5200-5205.

Rodriguez, M. A., \& Pepe, A. (2008). On the relationship between the structural and socioacademic communities of a coauthorship network. Journal of Informetrics, 2(3), 195-201.

Schummer, J. (2004). Multidisciplinarity, interdisciplinarity, and patterns of research collaboration in nanoscience and nanotechnology. Scientometrics, 59(3), 425-465.

Tarnow, E. (2002). Coauthorship in physics. Science and Engineering Ethics, 8(2), 175-190.

Tomassini, M., Luthi, L., Giacobini, M., \& Langdon, W. B. (2007). The structure of the genetic programming collaboration network. Genetic Programming and Evolvable Machines, 8(1), 97-103.

Traweek, S. (1992). Beamtimes and lifetimes: The world of high energy physicists. Cambridge, MA: Harvard University Press.

Wagner, C. S., \& Leydesdorff, L. (2005). Network structure, self-organization, and the growth of international collaboration in science. Research Policy, 34(10), 1608-1618.

Wasserman, S., \& Faust, K. (1994). Social network analysis. Cambridge: Cambridge University Press.

Watts, D. J., \& Strogatz, S. H. (1998). Collective dynamics of 'small-world' networks. Nature, 393(6684), 440-442. 